\title{
Prevalence of metabolically obese but normal weight (MONW) and metabolically healthy but obese (MHO) in Chinese Beijing urban subjects
}

\author{
Yan Zhang ${ }^{1}$, Jing Fu ${ }^{1}$, Shuwen Yang ${ }^{1}$, Ming Yang ${ }^{1}$, Annan Liu ${ }^{1}$, Leilei Wang ${ }^{1}$, Suyan Cao ${ }^{1, *}$, \\ Xue Sun ${ }^{1}$, Fang Wang ${ }^{2}$, Deping Liu ${ }^{2}$ \\ ${ }^{1}$ Department of VIP Medical Service, Beijing Hospital, National Center of Gerontology, Beijing, China; \\ ${ }^{2}$ Department of Cardiology, Beijing Hospital, National Center of Gerontology, Beijing, China.
}

\begin{abstract}
Summary The aim of this study was to assess the prevalence of metabolic syndrome (MetS) in nonobese adults (body mass index $(\mathrm{BMI})<25 \mathrm{~kg} / \mathrm{m}^{2}$ ) and the prevalence of obese adults (body mass index $(\mathrm{BMI}) \geq 25 \mathrm{~kg} / \mathrm{m}^{2}$ ) without MetS in Chinese Beijing urban subjects. A crosssectional study was conducted and the subjects who came to the hospital to receive a health examination were enrolled randomly. Regardless of age stratification, men have a higher prevalence of MetS than women. Among the urban Beijing population, prevalence of metabolically obese but normal weight (MONW) is lower than metabolically healthy but obese (MHO) regardless of gender. Except for the underweight group, participants exhibit significant differences between MetS and non-MetS subgroups in all tested variables in normal weight and overweight groups, whereas MONW and MHO participants exhibit significant differences in all variables except for creatinine (CR), aspartate aminotransferase (AST), uric acid (UAC) and high-density lipoprotein cholesterol (HDL-C). Women tend to have a higher MONW prevalence but lower MHO prevalence than men. Accordingly, MetS happens more frequently among those 40-59 yr. Besides, sex, age, WC, SBP, DBP, ALT, FG, UAC, TG, HDL-C and LDL-C are risk factors for MetS after multivariate adjustment. In conclusion, the prevalence of MONW is lower than MHO regardless of gender. Women tend to have a higher MONW prevalence but lower MHO prevalence than men.
\end{abstract}

Keywords: Metabolic syndrome, metabolically obese but normal weight (MONW), metabolically healthy but obese (MHO), prevalence

\section{Introduction}

Metabolic syndrome (MetS), also named as syndrome $\mathrm{X}$ or the insulin resistance syndrome, has existed as a public health issue for almost eight decades (1). Recently, with the change in dietary structure and the pervasiveness of diabetes and obesity, more people have been identified with MetS. In the United States, around $35 \%$ of adults and $50 \%$ of the elderly people (more than

Released online in J-STAGE as advance publication July 24, 2017.

*Address correspondence to:

Dr. Suyan Cao, Department of VIP Medical Service Ward D02, Beijing Hospital, National Center of Gerontology, No.1

Dahua Road, Dongdan, Beijing 100730, China.

E-mail: huxiaoqu57@163.com
60 years old) were estimated to have MetS during 2003 and 2012 (2). In China, the condition is similar to the United States and many other countries and areas (35). A series of studies have shown a relatively higher prevalence of MetS in urban areas than in rural areas nationwide. Until 2014, the MetS prevalence was much higher than that of decades ago (6).

The explicit definition of MetS was raised two decades ago by different organizations such as World Health Organization (WHO), the European Group for the Study of Insulin Resistance (EGSIR) etc. Although different definitions agree on the major essential categorical components like glucose intolerance and insulin resistance, small conflicts still exist (7). For example, both the definitions from WHO and EGSIR considered obesity or central obesity, while the American Association of Endocrinology suggests that obesity or 
central obesity should not be included in the identification process of MetS, because people with normal weight may also be insulin resistant. In 2004, experts from International Diabetes Federation (IDF) pointed out that the criteria of obesity used for MetS should be different in Asian areas compared to the western world since different areas may have diverse overweight incidences (8). This advice suggests that researchers should take geographical and racial differences into consideration when a MetS condition needs to be identified.

Metabolically obese but normal weight (MONW) and metabolically healthy but obese (MHO)are common and reveal different treatment effects in the clinic, notable benefits can be gained through a more comprehensive understanding of the prevalence of this subgroup population. MONW refers to the people who have normal body mass index but are related to increased levels of triglycerides, high blood pressure and the other characteristics of MetS (9). Among the population 20-40 years old, MONW may happen at the probability of $10-18 \%$ with a high incidence of cardiovascular diseases (10). To the contrary, individuals with MHO may reveal an unhealthy appearance, but they are usually metabolically healthy and have lower risk of cardiovascular diseases. Studies about the prevalence of MONW and MHO have been performed in western populations (2); however, research on Asian populations are limited. We herein collected a large amount of relevant data of the Beijing urban population to comprehensively acknowledge local prevalence of MONW and MHO.

\section{Materials and Methods}

\subsection{Study population}

A total of 22,376 subjects (13,748 men, 8,628 women, age from 18 to 85 ) were enrolled in this study from the health examination center of Beijing Hospital during January 2010 and December 2010. The enrolled subjects were randomly selected from all the individuals who came to the center for health examinations. Analyses of risk factors closely correlated with MetS was restricted to individuals who had a complete physical and biochemical measurements $(n=5,556)$. We attempted to study the detailed biochemical characteristics within the MetS groups so we only included MetS patients with complete physical and biochemical measurements. The reason for unfinished ones is that they did not have the relevant physical and biochemical measurements examinations. All participants signed informed consent, and the protocol was approved by China Health Statistics Center.

\subsection{Physical examination and biochemical analysis}

Physical examination and laboratory tests were performed as previously described $(11,12)$. Their blood pressure (BP) was measured three times and the average was used for analysis. BP was measured when the subject was in a seated position using a manual mercury sphygmomanometer. Weight and height of every individual were measured three times respectively during the physical examination. Weight was measured to the minimum $100 \mathrm{~g}$ with light clothing and without shoes, and height was measured to the minimum $1 \mathrm{~mm}$ without shoes. Waist circumference (WC) was measured to the minimum $1 \mathrm{~cm}$ at the navel level, and calculated as the average of one measurement after inspiration and one after expiration.

Overnight fasting blood samples were collected and analyzed using a Hitachi Modular DPE system (Roche Diagnostics, Penzberg, Germany). The plasma glucose level was measured using the hexokinase enzymatic method. Concentrations of biochemical molecules were analyzed using an auto-analyzer (Model 747-200, RocheHitachi).

\subsection{Definition of MetS, MONW, MHO, MHNW and $\mathrm{MOO}$}

The criteria we used to diagnose MetS were modified from those of the International Diabetes Federation (IDF) and the World Health organization-Asia Pacific region guideline, as having three or more of (1) WC $\geq$ $90 \mathrm{~cm}$ for male and $\geq 80 \mathrm{~cm}$ for female; (2) triglycerides (TG) concentration $\geq 150 \mathrm{mg} / \mathrm{dL}$; (3) high-density lipoprotein cholesterol (HDL-C) concentration $<40 \mathrm{mg} /$ $\mathrm{dL}$ for male and $<50 \mathrm{mg} / \mathrm{dL}$ for female or taking antihyperlipidemic medications; (4) $\mathrm{BP} \geq 130 / 85 \mathrm{mmHg}$ or taking antihypertensive medications; (5) fasting plasma glucose $>100 \mathrm{mg} / \mathrm{dL}$ or taking anti-diabetic medications, insulin or oral agents.

BMI was calculated as the ratio of weight in kilograms and the square of height in meters. According to WHO definitions, overweight was defined as BMI $\geq 25 \mathrm{~kg} / \mathrm{m}^{2}$. According to the relation between metabolically obesity and weight, participants of this study were divided into four categories: metabolically obese but normal weight (MONW), metabolically healthy and normal weight (MHNW), metabolically healthy but obese (MHO) and metabolically obese and obese (MOO).

\subsection{Statistical analysis}

This study was designed to provide relatively precise estimates of the urban Beijing population of the prevalence of MetS on gender by different age and BMI groups. Prevalence estimates were calculated for the overall urban Beijing population by 3 age groups or $3 \mathrm{BMI}$ groups. Besides, the prevalence estimates of MONW and MHO were calculated for men and women. Prevalence estimates between groups were compared 

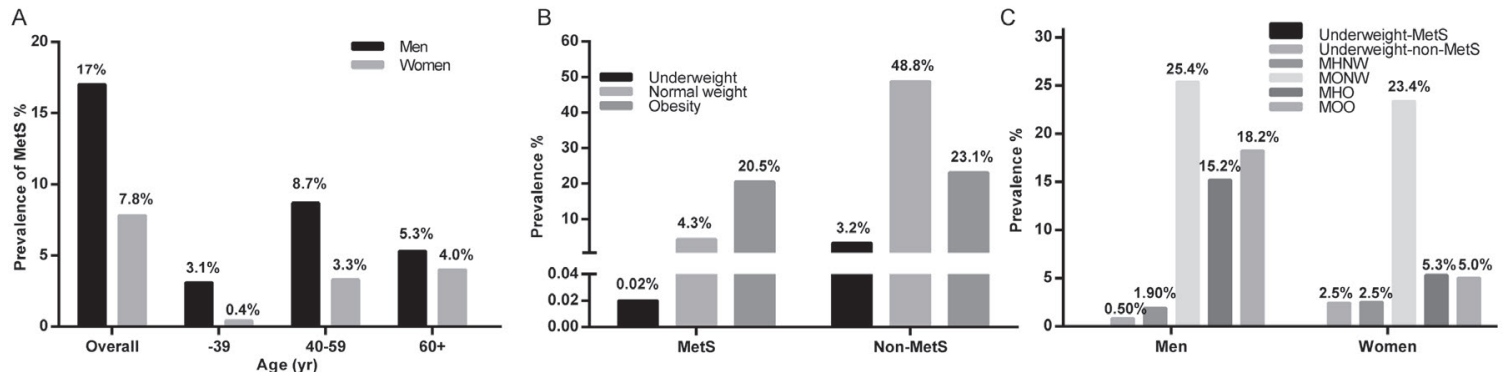

Figure 1. The prevalence of metabolic syndrome (MetS). A. The prevalence of MetS on gender by different age groups. Overall, Men had a much higher MetS prevalence than women did. Men had their highest MetS prevalence in their 40s and 50s but had their lowest MetS prevalence under 39. Women had the highest MetS prevalence in the $>60$ group but the lowest MetS prevalence under 39. B. The prevalence of MetS on BMI. Overall, most of the population was normal weight with no MetS, and normal weight with MetS (MONW) had higher prevalence than obesity with non-MetS (MHO) did. C. The prevalence of MetS on BMI and gender. Underweight people did not show MetS. Among the men and women, MONW demonstrated a higher prevalence than MHO.

Table 1. Prevalence statistics of metabolic syndrome

\begin{tabular}{|c|c|c|c|c|c|c|}
\hline \multirow{2}{*}{ Age (yr) } & \multicolumn{3}{|c|}{ Men } & \multicolumn{3}{|c|}{ Women } \\
\hline & Total & MetS & Prevalence rate $(\%)$ & Total & MetS & Prevalence rate $(\%)$ \\
\hline$\leq 39$ & 4267 & 685 & $16.0(3.1)$ & 2888 & 99 & $3.4(0.4)$ \\
\hline $40-59$ & 5871 & 1951 & $33.2(8.7)$ & 3829 & 738 & $19.3(3.3)$ \\
\hline$\geq 60$ & 3609 & 1177 & $32.6(5.3)$ & 1912 & 906 & $47.4(4.0)$ \\
\hline Total & 13747 & 3813 & $27.8(17.0)$ & 8629 & 1743 & $20.2(7.8)$ \\
\hline
\end{tabular}

MetS: metabolic syndrome.

using $\chi^{2}$ test. Statistical significance was met with a twotailed $P<0.05$. Statistical analyses were done using SPSS 20.0 (IBM, Armonk, NY, USA). Categorical variables were presented as numbers and percentages. Continuous variables were presented as mean \pm standard deviation (SD). After checking for the normality, continuous variables were analyzed using the Student's $t$-test. The differences between subgroups were analyzed using Student's $t$-test. The risk factor analysis was done using a univariate and multivariate logistic regression method.

\section{Results}

\subsection{The prevalence of MetS among the participants}

Approximately $24.8 \%$ of the participants have MetS (27.8\% of the males and $20.2 \%$ of the females have MetS). Overall, men had a much higher MetS prevalence than women did.

In males, the prevalence of MetS was 3.1\% among the $\leq 39 \mathrm{yrs}, 8.7 \%$ among the $40-59 \mathrm{yrs}$ and $5.3 \%$ among the $\geq 60$ yrs. Men had their highest MetS prevalence in their 40s and 50s but had their lowest MetS prevalence under 39 (Figure 1A, Table 1).

In females, the prevalence of MetS was $0.4 \%$ among the $\leq 39 \mathrm{yrs}, 3.3 \%$ among the $40-59 \mathrm{yrs}$ and $4.0 \%$ among the $\geq 60$ yrs (Figure 1A, Table 1). Women had the highest MetS prevalence in the $>60$ group but the lowest MetS prevalence under 39 (Figure 1A).

\subsection{Baseline characteristics of the participants}

The baseline characteristics of the study populations are presented in Table 2. The enrolled 22376 subjects consisted of 13,748 males (61.4\%) and 8,628 females (38.5\%). 3.3\% of the participants were underweight, $53.1 \%$ were normal weight and $43.6 \%$ were obese. Data for the 5556 MetS with complete physical and biochemical measurements are presented in Table S1 (http://www.biosciencetrends.com/action/ getSupplementalData.php?ID=11). The prevalence of non-MetS is 160-, 11.35- and 1.13-fold of that of MetS in underweight, normal weight and the obesity groups, respectively, suggesting that the obese participants have a higher risk of getting MetS. In addition, the distal blood pressure (DBP), TG, uric acid (UAC) and alanine aminotransferase (ALT) differed significantly between the non-MetS subgroup and the MetS subgroup within the underweight group. However, in the normal weight group, all variables except for gender and UAC differed substantially between the non-MetS and the MetS subgroups. Besides, in the overweight group, despite of gender, age, height, weight/height $(\mathrm{W} / \mathrm{H})$ ratio and creatinine (CR), all variables exhibited significant differences between the non-MetS subgroup and the MetS subgroup. Furthermore, when comparing the MONW and MHO subgroups, gender, age, HDL-C, UAC, CR and aspartate aminotransferase (AST) did not show a difference but the rest of the variables did, indicating that 'the rest of the variables' could possibly 


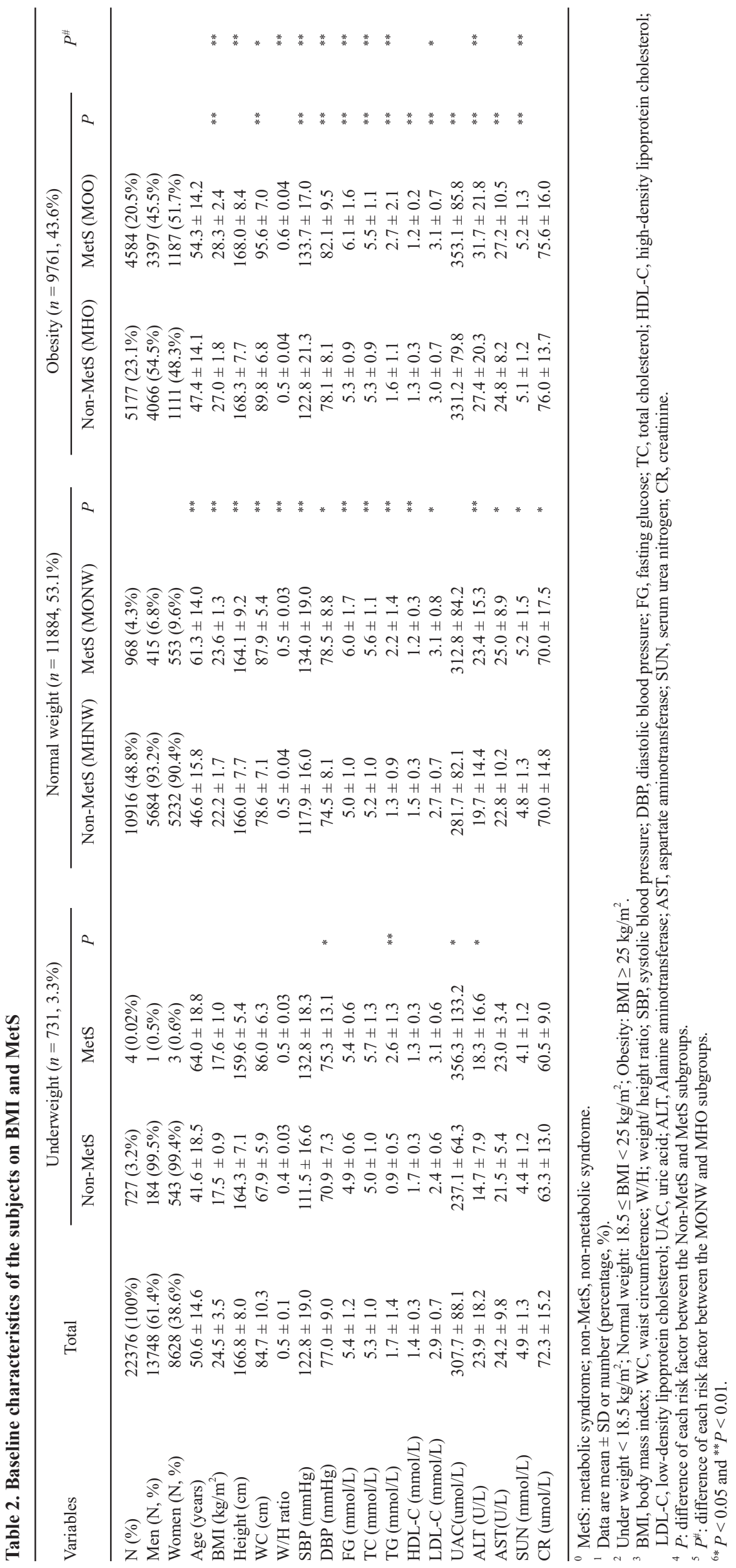


Table 3. Baseline characteristics of the subjects by gender, and BMI among the normal-weight

\begin{tabular}{|c|c|c|c|c|c|c|c|c|}
\hline \multirow{2}{*}{ Variables } & \multicolumn{2}{|c|}{ MHNW } & \multicolumn{2}{|c|}{ MONW } & \multirow{2}{*}{$P^{l}$} & \multirow{2}{*}{$P^{2}$} & \multirow{2}{*}{$P^{3}$} & \multirow{2}{*}{$P^{4}$} \\
\hline & Men & Women & Men & Women & & & & \\
\hline $\mathrm{N}(\%)$ & $5684(25.4 \%)$ & $5232(23.4 \%)$ & $415(1.90 \%)$ & $553(2.5 \%)$ & & & & \\
\hline BMI $\left(\mathrm{kg} / \mathrm{m}^{2}\right)$ & $22.72 \pm 1.6$ & $21.66 \pm 1.7$ & $23.87 \pm 1.1$ & $23.33 \pm 1.4$ & $* *$ & $* *$ & $* *$ & $* *$ \\
\hline Height $(\mathrm{cm})$ & $170.85 \pm 6.1$ & $160.60 \pm 5.4$ & $172.01 \pm 6.3$ & $158.18 \pm 56.0$ & & $* *$ & $* *$ & \\
\hline $\mathrm{WC}(\mathrm{cm})$ & $82.58 \pm 5.5$ & $74.23 \pm 6.0$ & $92.671 \pm 2.7$ & $84.31 \pm 4.0$ & $* *$ & $* *$ & $* *$ & $* *$ \\
\hline $\mathrm{W} / \mathrm{H}$ ratio & $0.48 \pm 0.03$ & $0.46 \pm 0.04$ & $0.54 \pm 0.02$ & $0.53 \pm 0.03$ & $* *$ & $* *$ & $* *$ & $* *$ \\
\hline $\mathrm{SBP}(\mathrm{mmHg})$ & $121.79 \pm 16.0$ & $113.73 \pm 15.0$ & $134.47 \pm 19.0$ & $133.60 \pm 19.0$ & $* *$ & $* *$ & $* *$ & \\
\hline $\mathrm{DBP}(\mathrm{mmHg})$ & $76.28 \pm 8.1$ & $72.57 \pm 7.7$ & $79.82 \pm 9.3$ & $77.50 \pm 8.2$ & $* *$ & & $*$ & $*$ \\
\hline $\mathrm{FG}(\mathrm{mmol} / \mathrm{L})$ & $5.33 \pm 1.2$ & $5.02 \pm 0.6$ & $6.11 \pm 1.6$ & $5.87 \pm 1.7$ & $* *$ & $* *$ & $* *$ & \\
\hline $\mathrm{TC}(\mathrm{mmol} / \mathrm{L})$ & $5.16 \pm 1.0$ & $5.17 \pm 1.0$ & $5.40 \pm 1.1$ & $5.72 \pm 1.1$ & & $* *$ & $* *$ & $*$ \\
\hline $\mathrm{TG}(\mathrm{mmol} / \mathrm{L})$ & $1.48 \pm 1.1$ & $1.13 \pm 0.6$ & $2.31 \pm 1.7$ & $2.14 \pm 1.0$ & $* *$ & $* *$ & $* *$ & $* *$ \\
\hline HDL-C (mmol/L) & $1.34 \pm 0.3$ & $1.58 \pm 0.3$ & $1.16 \pm 0.3$ & $1.28 \pm 0.3$ & $*$ & $* *$ & $* *$ & \\
\hline LDL-C $(\mathrm{mmol} / \mathrm{L})$ & $2.80 \pm 0.7$ & $2.63 \pm 0.7$ & $2.98 \pm 0.7$ & $3.12 \pm 0.8$ & & $*$ & & \\
\hline $\mathrm{UAC}(\mathrm{umol} / \mathrm{l})$ & $329.48 \pm 72.1$ & $229.75 \pm 56.9$ & $355.36 \pm 80.6$ & $280.79 \pm 72.0$ & $*$ & $* *$ & $* *$ & $*$ \\
\hline ALT (U/L) & $22.78 \pm 16.3$ & $16.33 \pm 11.1$ & $26.63 \pm 16.4$ & $20.98 \pm 14.0$ & & $* *$ & $* *$ & $*$ \\
\hline $\operatorname{AST}(\mathrm{U} / \mathrm{L})$ & $23.81 \pm 12.3$ & $21.60 \pm 6.9$ & $25.59 \pm 9.4$ & $24.57 \pm 8.6$ & & $* *$ & $* *$ & \\
\hline $\mathrm{SUN}(\mathrm{mmol} / \mathrm{L})$ & $5.17 \pm 1.3$ & $4.33 \pm 1.1$ & $5.46 \pm 1.4$ & $4.92 \pm 1.5$ & $*$ & $* *$ & $* *$ & \\
\hline $\mathrm{CR}(\mathrm{umol} / \mathrm{L})$ & $79.96 \pm 12.0$ & $59.05 \pm 8.6$ & $80.56 \pm 15.4$ & $62.12 \pm 14.6$ & $* *$ & $* *$ & $* *$ & $*$ \\
\hline
\end{tabular}

${ }^{0}$ MHNW: metabolically healthy and normal weight; MONW: metabolically obese but normal weight.

${ }^{1}$ Normal weight: $18.5 \leq \mathrm{BMI}<25 \mathrm{~kg} / \mathrm{m}^{2}$.

${ }^{2}$ BMI, body mass index; WC, waist circumference; W/H; weight/ height ratio; SBP, systolic blood pressure; DBP, diastolic blood pressure; FG, fasting glucose; TC, total cholesterol; HDL-C, high-density lipoprotein cholesterol; LDL-C, low-density lipoprotein cholesterol; UAC, uric acid; ALT, Alanine aminotransferase; AST, aspartate aminotransferase; SUN, serum urea nitrogen; CR, creatinine.

${ }^{3} P^{l}$ represents the difference of each risk factor for men between MHNW and MONW subgroups.

${ }^{4} P^{2}$ represents the difference of each risk factor for women between MHNW and MONW subgroups.

${ }^{5} P^{3}$ represents the difference of each risk factor between men and women in MHNW subgroup.

${ }^{6} P^{4}$ represents the difference of each risk factor between men and women in MONW subgroup.

${ }^{*} P<0.05$ and ${ }^{* *} P<0.01$

be the risk factors for the imbalance of metabolism and body weight. Overall, the prevalence of non-MetS is higher than MetS (Figure 1B), and women tend to have lower prevalence of MHNW, MONW, MHO and MOO (Figure 1C). Biochemical factors together with BMI index could be significant indicators of MetS.

\subsection{Characteristics by gender and BMI among the normal-weight subjects}

BMI, WC, W/H, SBP, DBP, FG, TG, HDL-C, UAC, SUN and CR are significantly different for men in the MHNW group from those in the MONW group. Whereas, among women, all variables except for DBP differ in the MHNW group and MONW group. In the MHNW group, men and women have significantly different levels of all variables except for LDL-C. On the other hand, men and women have significantly different levels of BMI, WC, W/H, DBP, TC, TG, UAC, ALT and CR in the MONW group (Table 3). It is worth mentioning that MONW participants showed higher mean values for all physical examination and laboratory test variables except for HDL-C compared to MHNW participants (MONW participants have lower HDL-C levels than MHNW counterparts). Details of underweight and obesity groups can be found in Table S2 (http://www.biosciencetrends.com/ action/getSupplementalData.php?ID=11). Data for the 5556 MetS in normal weight, underweight and obesity groups with complete physical and biochemical measurements are presented in Table S3 (http://www. biosciencetrends.com/action/getSupplementalData. php? ID=11).

\subsection{Characteristics of subjects by age among the non- MetS}

Overall, MONW participants had the highest prevalence in $60+$ yrs women $(28.29 \%)$ and men $(11.32 \%)$. MHO participants had a prevalence peak in the $\leq 39$ yrs age group (men, 69.15\% and women, 76.69\%) (Figure 2). The characteristics of study subjects stratified by age among non-MetS are presented in Table 4. In three age groups $(<40 \mathrm{yrs}, 40-59 \mathrm{yrs}$ and $>60 \mathrm{yrs})$, the overall prevalence of non-MetS was $37.9 \%, 41.67 \%$ and $20.43 \%$, respectively. BMI, FG, HDL-C, LDL-C, ALT, SUN, CR and UAC are significantly different between men and women in each age group. Height, WC, SBP, TC, TG and AST are significantly different between men and women in $\leq 39$ yrs group and 4059 yrs group. Detailed information in MetS and nonMetS groups across all age groups are shown in Table S4 (http://www.biosciencetrends.com/action/ getSupplementalData.php? ID=11). Data for the 5556 MetS with complete physical and biochemical measurements across three age groups are presented in Table S5 (http://www.biosciencetrends.com/action/ getSupplementalData.php?ID=11). 

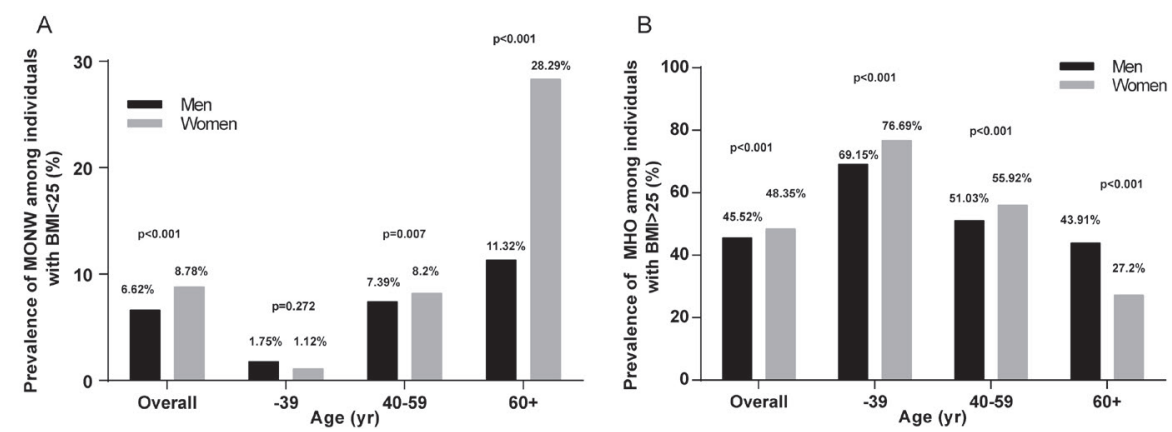

Figure 2. The prevalence of MONW and MHO. A. The prevalence of MONW among those with BMI $<25 \mathrm{~kg} / \mathrm{m}^{2}$ on age. Generally, women showed higher prevalence of MONW among the non-obese than men did. Both men and women had the highest prevalence of MONW in their 60 s and over. Both men and women showed the lowest prevalence in the $<39$ yrs group. B. The prevalence of $\mathrm{MHO}$ among the obese population by age. Overall, women demonstrated slightly higher prevalence of MHO than men did. Both male and female showed the highest prevalence in the $<39$ yrs group and the lowest prevalence in the $>60$ yrs group.

Table 4. Baseline characteristics of the subjects by gender and age among the non-MetS

\begin{tabular}{|c|c|c|c|c|c|c|c|c|c|}
\hline \multirow{2}{*}{ Variables } & \multicolumn{2}{|c|}{$\leq 39$ yrs } & \multirow{2}{*}{$P$} & \multicolumn{2}{|c|}{$40-59$ yrs } & \multirow{2}{*}{$P$} & \multicolumn{2}{|c|}{$\geq 60 \mathrm{yrs}$} & \multirow{2}{*}{$P$} \\
\hline & Men & Women & & Men & Women & & Men & Women & \\
\hline$N(\%)$ & $3586(21.32 \%)$ & $2789(16.58 \%)$ & & $3918(23.29 \%)$ & $3091(18.38 \%)$ & & $2430(14.45 \%)$ & $1006(5.98 \%)$ & \\
\hline BMI $\left(\mathrm{kg} / \mathrm{m}^{2}\right)$ & $24.3 \pm 3.0$ & $21.1 \pm 2.7$ & $* *$ & $24.9 \pm 2.6$ & $22.9 \pm 2.8$ & $* *$ & $23.7 \pm 2.8$ & $22.9 \pm 3.1$ & $* *$ \\
\hline Height $(\mathrm{cm})$ & $173.1 \pm 5.8$ & $162.1 \pm 5.3$ & $* *$ & $171.1 \pm 5.4$ & $160.5 \pm 5.0$ & $* *$ & $167.1 \pm 5.8$ & $156.0 \pm 5.7$ & \\
\hline $\mathrm{WC}(\mathrm{cm})$ & $84.9 \pm 7.5$ & $72.4 \pm 7.0$ & $*$ & $87.0 \pm 6.8$ & $76.8 \pm 7.1$ & $*$ & $85.5 \pm 7.8$ & $78.7 \pm 8.1$ & \\
\hline $\mathrm{W} / \mathrm{H}$ ratio & $0.5 \pm 0.04$ & $0.5 \pm 0.04$ & & $0.5 \pm 0.04$ & $0.5 \pm 0.1$ & & $0.5 \pm 0.1$ & $0.5 \pm 0.1$ & ** \\
\hline SBP (mmHg) & $117.4 \pm 11.1$ & $108.2 \pm 10.4$ & $*$ & $120.5 \pm 13.6$ & $115.1 \pm 24.5$ & $*$ & $133.3 \pm 18.4$ & $128.9 \pm 18.8$ & \\
\hline $\mathrm{DBP}(\mathrm{mmHg})$ & $76.1 \pm 7.3$ & $71.0 \pm 67.0$ & & $78.7 \pm 8.4$ & $73.9 \pm 8.0$ & & $76.8 \pm 8.8$ & $74.5 \pm 7.8$ & $* *$ \\
\hline $\mathrm{FG}(\mathrm{mmol} / \mathrm{L})$ & $5.0 \pm 0.6$ & $4.8 \pm 0.4$ & $* *$ & $5.4 \pm 1.1$ & $5.1 \pm 0.6$ & $* *$ & $5.7 \pm 1.5$ & $5.3 \pm 0.8$ & $* *$ \\
\hline $\mathrm{TC}(\mathrm{mmol} / \mathrm{L})$ & $5.0 \pm 0.9$ & $4.7 \pm 0.8$ & $* *$ & $5.3 \pm 0.9$ & $5.4 \pm 1.0$ & $* *$ & $5.3 \pm 1.0$ & $5.9 \pm 1.0$ & \\
\hline $\mathrm{TG}(\mathrm{mmol} / \mathrm{L})$ & $1.5 \pm 1.2$ & $0.9 \pm 0.4$ & $* *$ & $1.7 \pm 1.1$ & $1.2 \pm 0.6$ & $* *$ & $1.4 \pm 0.7$ & $1.5 \pm 0.7$ & \\
\hline HDL-C (mmol/L) & $1.3 \pm 0.3$ & $1.6 \pm 0.3$ & $* *$ & $1.3 \pm 0.3$ & $1.6 \pm 0.3$ & $* *$ & $1.4 \pm 0.3$ & $1.6 \pm 0.3$ & $* *$ \\
\hline LDL-C (mmol/L) & $2.8 \pm 0.7$ & $2.3 \pm 0.6$ & $* *$ & $3.0 \pm 0.7$ & $2.8 \pm 0.7$ & $* *$ & $2.8 \pm 0.7$ & $3.0 \pm 0.7$ & ** \\
\hline UAC(umol/L) & $338.6 \pm 70.2$ & $223.6 \pm 51.4$ & $* *$ & $335.4 \pm 68.8$ & $232.2 \pm 56.1$ & $* *$ & $340.2 \pm 82.5$ & $263.8 \pm 71.9$ & $* *$ \\
\hline ALT (U/L) & $28.0 \pm 21.7$ & $14.7 \pm 9.4$ & $* *$ & $26.4 \pm 19.0$ & $18.3 \pm 12.7$ & $* *$ & $19.86 \pm 11.62$ & $17.45 \pm 9.34$ & $*$ \\
\hline $\operatorname{AST}(\mathrm{U} / \mathrm{L})$ & $24.4 \pm 9.1$ & $20.4 \pm 5.4$ & $* *$ & $24.9 \pm 13.6$ & $22.5 \pm 7.6$ & $* *$ & $23.66 \pm 7.69$ & $23.31 \pm 6.78$ & \\
\hline SUN (mmol/L) & $4.9 \pm 1.1$ & $4.0 \pm 0.9$ & $* *$ & $5.1 \pm 1.1$ & $4.5 \pm 1.1$ & $* *$ & $5.64 \pm 1.43$ & $5.16 \pm 1.19$ & $* *$ \\
\hline CR (umol/L) & $79.31 \pm 9.3$ & $57.49 \pm 7.34$ & $* *$ & $79.31 \pm 10.28$ & $59.13 \pm 8.33$ & $* *$ & $82.56 \pm 15.78$ & $63.75 \pm 11.45$ & ** \\
\hline
\end{tabular}

${ }^{1}$ Data are mean \pm SD or number (percentage, \%).

${ }^{2}$ Non-MetS, non-metabolic syndrome; BMI, body mass index; WC, waist circumference; W/H; weight/ height ratio; SBP, systolic blood pressure; DBP, diastolic blood pressure; FG, fasting glucose; TC, total cholesterol; HDL-C, high-density lipoprotein cholesterol; LDL-C, low-density lipoprotein cholesterol; UAC, uric acid; ALT, Alanine aminotransferase; AST, aspartate aminotransferase; SUN, serum urea nitrogen; CR, creatinine. ${ }^{3} P$ represents the difference of each risk factor for Men and Women in Non-MetS subgroups

${ }^{*} P<0.05$ and ${ }^{* *} P<0.01$

\subsection{Risk factor analysis of MetS}

Logistic regression analysis was done to analyze the risk factors of MetS. The results are shown in Table 5. The results demonstrate that sex, age, WC, SBP, DBP, ALT, FG, UAC, TG, HDL-C and LDL-C are all very significant risk factors for MetS.

\section{Discussion}

Our results revealed that not only obese but nonobese individuals in Beijing urban population also had metabolism-associated disorders. MONW is less common than MHO among all participants regardless of gender. Males had higher MHO incidence but lower MONW incidence than females. Age is associated with other variables. Males aged between 40-59 yrs had the highest MetS prevalence compared to those aged above 60 yrs or under 39 yrs. Among females, the highest MetS prevalence is present after age 60 , which may be due to postmenopausal status $(13,14)$.

The worldwide MetS prevalence among adults ranges from $10 \%$ to $55 \%$, depending on the ethnic group, urbanization, lifestyle and diagnostic criteria (15-18). For instance, in China, it was estimated that the overall prevalence of MetS in adults was $11.1 \%$ in 1991 and $26.1 \%$ in 2004 . The changes of MetS prevalence across the last two decades suggest that the prevalence of MetS has become higher with the development of urbanization. From another study in 2011, Uygur ethnic group has a higher prevalence of MetS than the Han group because of high intake of animal fats, proteins 
Table 5. Univariate and multivariate logistic regression analysis of MetS

\begin{tabular}{|c|c|c|c|c|}
\hline \multirow{2}{*}{ Variables } & \multicolumn{2}{|c|}{ Univariate } & \multicolumn{2}{|c|}{ Multivariate } \\
\hline & OR $(95 \% \mathrm{CI})$ & $P$ value & OR $(95 \% \mathrm{CI})$ & $P$ value \\
\hline Sex & $1.516(1.422,1.617)$ & $<0.001$ & $0.053(0.044,0.065)$ & $<0.001$ \\
\hline Age (yrs) & $1.037(1.035,1.039)$ & $<0.001$ & $1.024(1.019,1.029)$ & $<0.001$ \\
\hline $\mathrm{WC}(\mathrm{cm})$ & $1.199(1.193,1.206)$ & $<0.001$ & $1.245(1.229,1.260)$ & $<0.001$ \\
\hline Height (cm) & $1.012(1.008,1.016)$ & $<0.001$ & $1.003(0.991,1.015)$ & 0.616 \\
\hline Weight & $1.066(1.063,1.069)$ & $<0.001$ & $0.998(0.990,1.007)$ & 0.676 \\
\hline BMI $\left(\mathrm{kg} / \mathrm{m}^{2}\right)$ & $1.549(1.528,1.570)$ & $<0.001$ & $1.023(0.988,1.059)$ & 0.207 \\
\hline SBP (mmHg) & $1.050(1.048,1.052)$ & $<0.001$ & $1.025(1.021,1.029)$ & $<0.001$ \\
\hline DBP (mmHg) & $1.082(1.078,1.086)$ & $<0.001$ & $1.034(1.026,1.042)$ & $<0.001$ \\
\hline $\operatorname{ALT}(\mathrm{U} / \mathrm{L})$ & $1.026(1.024,1.028)$ & $<0.001$ & $1.004(1.000,1.008)$ & 0.041 \\
\hline AST (U/L) & $1.046(1.042,1.050)$ & $<0.001$ & $1.005(0.997,1.014)$ & 0.186 \\
\hline $\mathrm{FG}(\mathrm{mmol} / \mathrm{L})$ & $2.043(1.968,2.120)$ & $<0.001$ & $1.455(1.392,1.521)$ & $<0.001$ \\
\hline SUN (mmol/L) & $1.242(1.213,1.271)$ & $<0.001$ & $0.967(0.925,1.010)$ & 0.129 \\
\hline CR (umol/L) & $1.013(1.011,1.015)$ & $<0.001$ & $1.002(0.997,1.007)$ & 0.39 \\
\hline UAC(umol/L) & $1.007(1.006,1.007)$ & $<0.001$ & $1.002(1.001,1.002)$ & $<0.001$ \\
\hline $\mathrm{TG}(\mathrm{mmol} / \mathrm{L})$ & $2.522(2.431,2.616)$ & $<0.001$ & $1.618(1.538,1.702)$ & $<0.001$ \\
\hline HDL-C (mmol/L) & $0.032(0.028,0.037)$ & $<0.001$ & $0.051(0.040,0.065)$ & $<0.001$ \\
\hline LDL-C (mmol/L) & $1.781(1.702,1.863)$ & $<0.001$ & $1.181(1.101,1.267)$ & $<0.001$ \\
\hline
\end{tabular}

BMI, body mass index; WC, waist circumference; SBP, systolic blood pressure; DBP, diastolic blood pressure; FG, fasting glucose; HDL-C, high-density lipoprotein cholesterol; LDL-C, low-density lipoprotein cholesterol; UAC, uric acid; ALT, Alanine aminotransferase; AST, aspartate aminotransferase; SUN, serum urea nitrogen; CR, creatinine.

and salts and less exercise. Besides, the prevalence of MetS in Caucasians was higher than that in Asia (19). These studies confirmed that ethnic and life styles could contribute to the differences of MetS prevalence. We aimed to study the prevalence of MHO and MONW among the population in Beijing city only to comprehend the understanding of the current situation of MetS and obesity. In this study, we found that sex, age, WC, SBP, DBP, ALT, FG, UAC, TG, HDL-C and LDL-C are all very significant risk factors for MetS. With increase in age, the medial layer of the vessel wall appears gradually degenerated, the middle collagen content increases and the elastic layer fractures. With long-term hypertension, the structural change is more obvious and intensified, because the large artery stiffness exacerbates with aging, flexibility also declines with a high risk of vascular disease. In addition, our results showed that males between 40-59 yrs were prone to have MetS whereas females above 60 yrs were more likely to have MetS, suggesting that MetS is a serious public health burden affecting people in Beijing.

Having known the high prevalence of MONW and MHO among the Beijing urban population, we wanted to see which factors are closely correlated with MetS. Physical and blood examinations were both done to uncover the correlation between MetS and variables. UAC is the product of purine metabolism in humans and MetS individuals often have high UAC levels; however, the association of UAC levels and the prevalence of MetS remains contradictory (2023). Little information on its association with MetS in Chinese population is available. Consistent with the previous finding, Pearson's correlation analyses in this work suggests that hyperuricemia is more correlated with MetS in males than in females (24). Our findings also suggested that UAC and TG could be a risk factor for MetS. Previous studies showed that high TG level was associated with hyperuricemia $(24,25)$. The possible explanation is that TG could promote the production of UAC and the synthesis of ribose-5phosphate to phosphoribosyl pyrophosphate (PPRP) (26). The UAC level was found closely associated with MetS incidence among the Beijing urban population; however, the precise mechanism underlying the association of UAC with MetS has not been elucidated and further studies are needed.

Some epidemiological research has shown that ALT and AST with MetS risk factors BMI, DBP, TG, HDL-C, LDL-C and UAC $(27,28)$. Previous studies suggest that high ALT levels are related to MetS and obesity in Japanese (27), Chinese (29) and Korean adolescents (30). The reason might be that ALT and AST are involved in fat accumulation in the liver and are closely correlated with fatty liver disease $(27,31,32)$. Intriguingly, we also found that ALT was more closely associated with MetS than AST among our subjects; however, the underlying mechanism explaining this finding remains to be further explored.

There are limitations to the present study that warrant further research. First of all, the study population only consists of the Beijing urban population, so it cannot represent the prevalence of MetS in Beijing suburban areas or other provinces. Secondly, this study did not include the influence of ethnic or lifestyles on MetS prevalence, which have been indicated to have effects on MetS prevalence.

In summary, we conducted this cross-sectional 
study to explore the prevalence of MONW and MHO among the Beijing urban population. The definition of MONW, MHO and MetS were made properly, therefore our findings could provide useful information for comprehension of the current situation of obesity and MetS. The prevalence of overweight and MetS in Beijing urban adults is dramatically high. Our findings provided useful information for the projection of future trends and developing national strategies and programs to address the challenges from the growing obesity and MetS.

\section{Acknowledgements}

The work is funded by China's 12th Five Year Plan Projects (2012ZX09303-008-002) and The National Natural Science Foundation (No. 51672030).

\section{References}

1. Kaur J. A comprehensive review on metabolic syndrome. Cardiol Res Pract. 2014; 2014:943162.

2. Aguilar M, Bhuket T, Torres S, Liu B, Wong RJ. Prevalence of the metabolic syndrome in the United States, 2003-2012. JAMA. 2015; 313:1973-1974.

3. Okafor CI. The metabolic syndrome in Africa: Current trends. Indian J Endocrinol Metab. 2012; 16:56-66.

4. Shen J, Goyal A, Sperling L. The emerging epidemic of obesity, diabetes, and the metabolic syndrome in china. Cardiol Res Pract. 2012; 2012:178675.

5. Mozumdar A, Liguori G. Persistent increase of prevalence of metabolic syndrome among U.S. adults: NHANES III to NHANES 1999-2006. Diabetes Care. 2011; 34:216-219.

6. Jiang B, Li B, Wang Y, Han B, Wang N, Li Q, Yang W, Huang G, Wang J, Chen Y, Chen Y, Zhu C, Lin $\mathrm{D}, \mathrm{Lu} \mathrm{Y}$. The nine-year changes of the incidence and characteristics of metabolic syndrome in China: longitudinal comparisons of the two cross-sectional surveys in a newly formed urban community. Cardiovasc Diabetol. 2016; 15:84.

7. Eckel RH, Grundy SM, Zimmet PZ. The metabolic syndrome. Lancet. 2005; 365:1415-1428.

8. Alberti KG, Eckel RH, Grundy SM, et al. Harmonizing the metabolic syndrome: a joint interim statement of the International Diabetes Federation Task Force on Epidemiology and Prevention; National Heart, Lung, and Blood Institute; American Heart Association; World Heart Federation; International Atherosclerosis Society; and International Association for the Study of Obesity. Circulation. 2009; 120:1640-1645.

9. Lopez-Miranda J, Perez-Martinez P. It is time to define metabolically obese but normal-weight (MONW) individuals. Clin Endocrinol (Oxf). 2013; 79:314-315.

10. Conus F, Rabasa-Lhoret R, Peronnet F. Characteristics of metabolically obese normal-weight (MONW) subjects. Appl Physiol Nutr Metab. 2007; 32:4-12.

11. Tsai $\mathrm{CH}$. Metabolic syndrome in non-obese Taiwanese: new definition of metabolically obese, normal-weight individual. Chin Med J (Engl). 2009; 122:2534-2539.

12. Kim MC, Kim CS, Chung TH, Jeong J, Lee SH, Kim SR, Jung SW, Park NH, Yoo CI. MONW phenotype is associated with advanced colorectal adenoma in Korean men. Obesity (Silver Spring). 2012; 20:1876-1881.

13. Lim H, Nguyen T, Choue R, Wang Y. Sociodemographic disparities in the composition of metabolic syndrome components among adults in South Korea. Diabetes care. 2012; 35:2028-2035.

14. Kim HM, Park J, Ryu SY, Kim J. The effect of menopause on the metabolic syndrome among Korean women: the Korean National Health and Nutrition Examination Survey, 2001. Diabetes care. 2007; 30:701706.

15. Nestel P, Lyu R, Low LP, Sheu WH, Nitiyanant W, Saito I, Tan CE. Metabolic syndrome: recent prevalence in East and Southeast Asian populations. Asia Pac J Clin Nutr. 2007; 16:362-367.

16. Eapen D, Kalra GL, Merchant N, Arora A, Khan BV. Metabolic syndrome and cardiovascular disease in South Asians. Vasc Health Risk Manag. 2009; 5:731-743.

17. Jain SR, Shah KH, Acharya HN, Barot K, Sharma KH. Prevalence and Predictors of Metabolic Syndrome in Young Asymptomatic Gujarati Population. Int J Chronic Dis. 2015; 2015:365217.

18. Wang X, Yang F, Bots ML, Guo WY, Zhao B, Hoes AW, Vaartjes I. Prevalence of the Metabolic Syndrome Among Employees in Northeast China. Chin Med J (Engl). 2015; 128:1989-1993.

19. Chen BD, Yang YN, Ma YT, Pan S, He CH, Liu F, Ma X, Fu ZY, Li XM, Xie X, Zheng YY. Waist-to-Height Ratio and Triglycerides/High-Density Lipoprotein Cholesterol Were the Optimal Predictors of Metabolic Syndrome in Uighur Men and Women in Xinjiang, China. Metab Syndr Relat Disord. 2015; 13:214-220.

20. Conen D, Wietlisbach V, Bovet P, Shamlaye C, Riesen W, Paccaud F, Burnier M. Prevalence of hyperuricemia and relation of serum uric acid with cardiovascular risk factors in a developing country. BMC public health. 2004; 4:9.

21. Desai MY, Santos RD, Dalal D, Carvalho JA, Martin DR, Flynn JA, Nasir K, Blumenthal RS. Relation of serum uric acid with metabolic risk factors in asymptomatic middleaged Brazilian men. Am J Cardiol. 2005; 95:865-868.

22. Hikita M, Ohno I, Mori Y, Ichida K, Yokose T, Hosoya $\mathrm{T}$. Relationship between hyperuricemia and body fat distribution. Intern Med. 2007; 46:1353-1358.

23. Zhang Q, Zhang C, Song X, Lin H, Zhang D, Meng W, Zhang Y, Zhu Z, Tang F, Liu L, Yang X, Xue F. A longitudinal cohort based association study between uric acid level and metabolic syndrome in Chinese Han urban male population. BMC public health. 2012; 12:419.

24. Nejatinamini S, Ataie-Jafari A, Qorbani M, Nikoohemat S, Kelishadi R, Asayesh H, Hosseini S. Association between serum uric acid level and metabolic syndrome components. J Diabetes Metab Disord. 2015; 14:70.

25. Clausen JO, Borch-Johnsen K, Ibsen H, Pedersen O. Analysis of the relationship between fasting serum uric acid and the insulin sensitivity index in a populationbased sample of 380 young healthy Caucasians. Eur J Endocrinol. 1998; 138:63-69.

26. Matsuura F, Yamashita S, Nakamura T, Nishida M, Nozaki S, Funahashi T, Matsuzawa Y. Effect of visceral fat accumulation on uric acid metabolism in male obese subjects: visceral fat obesity is linked more closely to overproduction of uric acid than subcutaneous fat obesity. Metabolism. 1998; 47:929-933.

27. Saito T, Nishise Y, Makino N, et al. Impact of metabolic 
syndrome on elevated serum alanine aminotransferase levels in the Japanese population. Metabolism. 2009; 58:1067-1075.

28. Liangpunsakul S, Chalasani N. Unexplained elevations in alanine aminotransferase in individuals with the metabolic syndrome: results from the third National Health and Nutrition Survey (NHANES III). Am J Med Sci. 2005; 329:111-116.

29. Hong Z, Yanfang J, Shumei H, Jie S, Qing G, Xiangwei F, Bing D, Wei W, Xiaodong S, Chunyan W, Siqi Z, Wanyu L, Junyan F, Junqi N. Relationship between serum aminotransferase levels and metabolic disorders in northern China. Turk J Gastroenterol. 2012; 23:699-707.

30. Park HS, Han JH, Choi KM, Kim SM. Relation between elevated serum alanine aminotransferase and metabolic syndrome in Korean adolescents. Am J Clin Nutr. 2005; 82:1046-1051.

31. Chen ZW, Chen LY, Dai HL, Chen JH, Fang LZ. Relationship between alanine aminotransferase levels and metabolic syndrome in nonalcoholic fatty liver disease. J Zhejiang Univ Sci B. 2008; 9:616-622.

32. Miyatake N, Matsumoto S, Makino H, Numata T. Comparison of hepatic enzymes between Japanese men with and without metabolic syndrome. Acta Med Okayama. 2007; 61:31-34.

(Received January 20, 2017; Revised April 24, 2017; Rerevised June 22, 2017; Accepted July 5, 2017) 